\title{
Physicochemical study of 3,4-dichlorophenoxyacetic acid intercalated into hydrotalcite- like compound by ion exchanged method
}

\begin{abstract}
The intercalation of herbicide, 3,4-dicholorophenoxyacetic acid (3,4D), into zinc-aluminiumlayered double hydroxide $(\mathrm{LDH})$ for the formation of a new nanocomposite ZADX, was accomplished via anion exchange method. Due to the intercalation of 3,4D with LDH interlayer domain, basal spacing expanded from $8.9 \AA$ in the ZAL to 17.7-19.0 $\AA$ in the ZADX. The percentage loading of 3,4D in the ZADX is $51.4 \%(\mathrm{w} / \mathrm{w})$. The FTIR spectra of the nanocomposite shows resemblance peaks of the 3,4D and Zn-Al-layered double hydroxide indicating the inclusion of 3,4D into the layered double hydroxide. Surface area of the resulting nanocomposite increased from 1.3 to $7.14 \mathrm{~m} 2 \mathrm{~g}-1$ with the nitrogen adsorptiondesorption of type IV.
\end{abstract}

Keyword: 3,4-dichlorophenoxyaceticacid; Herbicide; Hydrotalcite-like compound; Intercalation 